\title{
The Formal Method of Metaphor Understanding within Dynamic Context
}

\author{
Min Zhu ${ }^{1,2, a}$ \\ ${ }^{1}$ School of Philosophy and Social Development, Shandong University, \\ Jinan, 250100, China \\ ${ }^{2}$ School of Marxism, Harbin University of Science and Technology, \\ Harbin, 150080, China \\ a email: zm1127@sina.com
}

\begin{abstract}
The research of metaphor understanding is a significant field of cognitive science. Metaphor plays an important role in natural language discourse. There is a close relationship between logic and metaphor. Metaphor can be described by dynamic epistemic logic. This article, we attempt to dynamically describe the process of metaphor understanding from the perspective of formal logic. First, speaker need to put forward metaphorical proposition by announcement, at the same time to update the old context set. After listener heard the announcement of the speaker, he understands it, then announce his understanding as a proposition. Finally, some discussions about this logical system are showed.
\end{abstract}

Keywords: metaphor understanding; dynamic context; formalize; knowledge

\section{Introduction}

In the process of language communication, context and understanding are actually dynamic. So they cannot merely be regarded as a static process. As the language communication is processed, context set will be continuously extended or modified, understanding will be changed. For these which one is misunderstood before maybe understood now. The earlier understanding conclusion may be overturned, but a new understanding conclusion is produced. Therefore, we should consider the process of metaphor understanding in the dynamic context. Hope that we can restore the understanding process with integrity from the perspective of dynamic epistemic logic.

Logical expression can express the meaning of the proposition. Then proposition's meaning can process meaning of the sentence to a truth-value condition of the proposition. The true value can be calculated, but its calculation process must be conducted in the context. It depends on context and is restricted by context. A context set is composed of all public background knowledge necessary for communication. The propositions that dialogue with participants, believe "of course true", also can make up this set of background knowledge. In fact, public knowledge also can be seen as that we believe it is accurate or know 
it is a true proposition. When the speaker said in a sentence, he always has been presupposed 'he believes that this sentence is true'. Therefore, the public knowledge of the two communicative sides is the beliefs and the propositions which they all have or they can realize that they all have. In the dialogue, the new assertions, propositions, or beliefs join in the old context set will make the entire context set to simplify. Because all cases that are not consistent with the original assertions, propositions, or beliefs will be out of the context set. So the range of this context set maybe get to reduce and simplify. Note that the new information entering context set is not the sufficient condition for the change of context set or the necessary condition for the change of the context set. They do not necessarily change the public background knowledge of language communication. And there is a kind of situation that no new information joins but public knowledge is changed.

The old context set $\mathrm{C}$ is the set of background knowledge which both communicators have in current discourse. It usually contains more than one proposition that some agents think as true. And it will constantly add or update with the progress of the dialogue communication process. Note that: firstly, update of context set is created by the two communicative sides in communication process. Only when the speaker's knowledge and belief consist with the listener's knowledge and belief, the intersection between them will be as a new element added to the original context set $\mathrm{C}$. Then it becomes a part of the contemporary public background knowledge. Secondly, the new context set is a local context for the current communicators. When we include the words in context, we can't use all the context knowledge which cognitive agents have. Only the context that has a direct relationship with the current discourse understanding will be invoked.

In this paper, we attempt to dynamically describe the process of metaphor understanding from the perspective of formal logic. The context set can be seen as a possible world subset. It contains many characters and propositions. The dialogue can be viewed as a kind of public announcement. In this way, the dialogue behavior will proceed with the precise structure orderly. First of all, discourse participants announce the metaphor sentences as proposition, and cognitive context changes with the addition of new information and update. At the same time, after the listener knows this announcement, he will understand this proposition based on the context background knowledge, and then announces his own understanding. Finally, a set of dialogue goes smoothly. The continuous process of dialogue can be viewed as the superposition of the announcement.

\section{The Metaphorical Discourse Announcement by Speaker}

When the speaker announces a metaphor proposition, this behavior itself has the capacity to set up a public knowledge background, and has an effect to update the cognitive affection of current context set. Listener hears the contemporary metaphor discourse will unconsciously get that fact and understand it. Then he knows the meaning that the speaker wishes to convey. From the speaker to the 
listener is a process of dynamic transformation. External behavior caused the agent and the object to update their knowledge. Public announcement logic can well describe this basic cognitive behavior. Proposition he identifies is not a person's belief or knowledge, but the knowledge or belief of a certain group. We call this kind of belief or knowledge for the common belief or the public knowledge. The public knowledge can also be represented by logic.

In fact, public announcement logic language is added some modal operators which represent dynamic behavior on the static cognitive logic language. Based on this, we can add some definition to describe the metaphorical discourse announcement by speaker.

Definition1 (Logical language $\left.L_{K C[}\right](N, P)$ ) Given a finite set of agents $\mathrm{N}$ and a countable set of atoms P. Based on the public announcement logical language $\left.L_{K[}\right](N, P)$, logical language $L_{K C[}(N, P)$ which added public knowledge and metaphor, is inductively defined by,

$\varphi:=\mathrm{p}|\neg \varphi|(\varphi \wedge \psi)\left|\mathrm{K}_{i} \varphi\right|[\varphi] C \psi$

If $\mathrm{N}$ and $\mathrm{P}$ is not relevant, $\left.L_{K C[}\right](N, P)$ can be represented as $L_{K C[}$ ] ( ).

$[\varphi] C \psi$ means after announce $\varphi$ success, $\psi$ becomes public knowledge. Public announcement as an action is a partial function. It has the same meaning with "if successfully announce $\varphi$, it holds that $C \psi$ ".

Definition2 (Cognitive modal) Given a finite set of agents $\mathrm{N}$ and a countable set of atoms $\mathrm{P}$, the model of cognitive language $\left.L_{K[}\right](N, P)$ is a structure $\mathrm{M}=\left(W, D, C, R, R^{\prime}, V\right)$, where,

(1)W is a set of possible world, which is not $\emptyset$.

(2) $R$ is a function, from $\mathrm{N}$ to $2^{w^{* w}}$. Every agent in $\mathrm{N}$ is assigned a binary equivalent relation on the W. We always use $(R w t)$ to represent accessibility relation between $w$ and $t$.

(3) $V$ is a $\mathrm{P} \rightarrow 2^{W}$ truth function. It assigns every atomic proposition $p \in \mathrm{P}$ in every possible world.

(4) Every possible world $w$ is assigned an individual domain $D_{w}$ of a domain function D.

(5) $\mathrm{C}$ is a context set, which has various properties of the objects in the set of formulas. It is consistent. $C \subset W$, every context in $C$ is the subset of the possible world set $\mathrm{W}$. In other word, every context is a possible world.

(6) $R^{\prime}$ is a function, describes the accessibility relationship just in C. Main objective is to illustrate the similar relationship between $\mathrm{S}$ and $\mathrm{T}$ of the metaphorical propositions in context. $R^{f}$ is equivalent.

Definition3 (Semantics) Given a cognitive model $\mathrm{M}=\left(W, D, C, R, R^{v}, V\right)$, a finite set of agents $\mathrm{N}$ and a countable set of atoms P. In cognitive language $L_{K C[}$ ] ( ), each formula's true value can 
be inductively defined by,

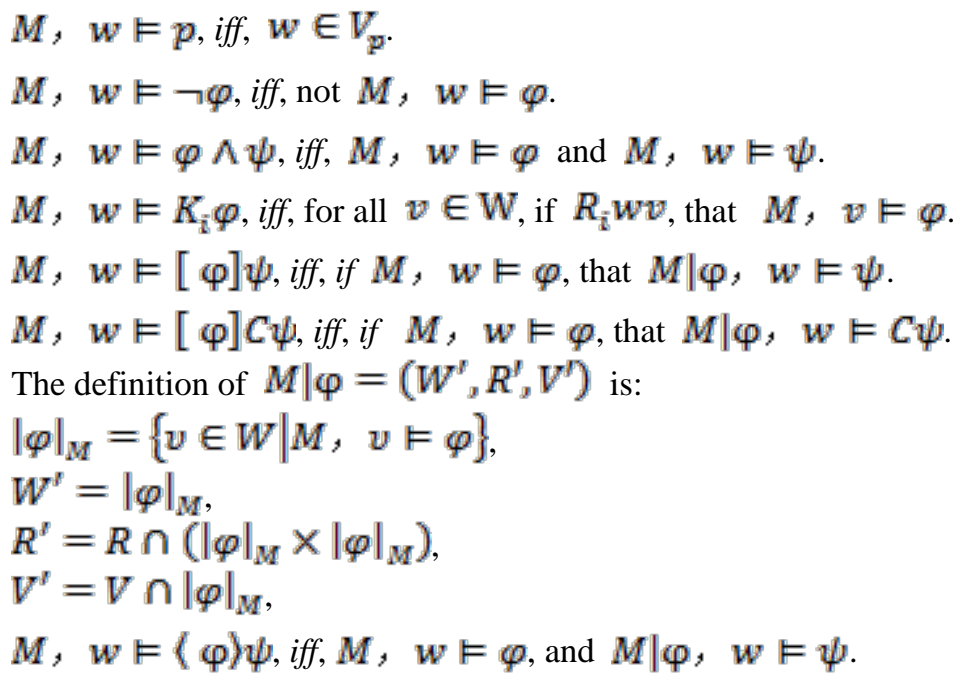

A point model $(M, w)$ can be viewed as a cognitive state. The public announcement proposition $\varphi$ changes the current knowledge state of the agent. It triggers the current change of agent from formal, $\psi$ becomes public knowledge which does not include the incompatible world with $\varphi$. It gets a new context set different with announcement not happen. Therefore, we can be said that the active modal operator $[\varphi]$ dynamically transforms a model to its relative model.

\section{The Public Announcement of Listener}

For the speaker, before he announced a metaphor proposition, the understanding of this metaphor proposition was already presupposed, and the context conditions needed to understand that this metaphor proposition also was presupposed. As a result, the work of listener agrees to the speaker's announcement, updates his context, understands the meaning of the metaphor proposition expressed, and announces his new understanding. All these help the speech communication continuous.

Difinition1 (Language $\left.L_{K C[}\right](N, P)$ ) Given a finite set of agents $\mathrm{N}$ and a countable set of atoms $\mathrm{P}$, logical language $\left.L_{K C[}\right](N, P)$ which added public knowledge and metaphor, is inductively defined by,

$$
\varphi:=\mathrm{p}|\neg \varphi|(\varphi \wedge \psi)\left|\mathrm{K}_{i} \varphi\right| \mathrm{C}_{B} \varphi \mid[\varphi] \psi\left(S, T, f_{M}\right)
$$

If $\mathrm{N}$ and $\mathrm{P}$ is not relevant, $\left.L_{K C[}\right](N, P)$ can be represented as $\left.L_{K C[}\right]($ ). 
$[\varphi] \psi\left(S, T, f_{M}\right)$ means after announce a metaphor proposition $\varphi$ succeed, by an analogical function $f_{M}, \psi$ can get the similarity between tenor (T) and vehicle (S), $\psi$ is the express meaning of metaphorical proposition. Because ' $\varphi$ is true' is the premise of this public announcement, proposition $\varphi$ must be metaphor sentence.

The cognitive semantic model of listener is consistent with the speaker's. It gives the semantics to listener.

Difinition2 (Semantics) Given a cognitive model $\mathrm{M}=\left(W, D, C, R, R^{\prime}, V\right)$, a finite set of agents $\mathrm{N}$ and a countable set of atoms P. In cognitive language $L_{K C[}$ ] ( ), each formula's true value can be inductively defined by,

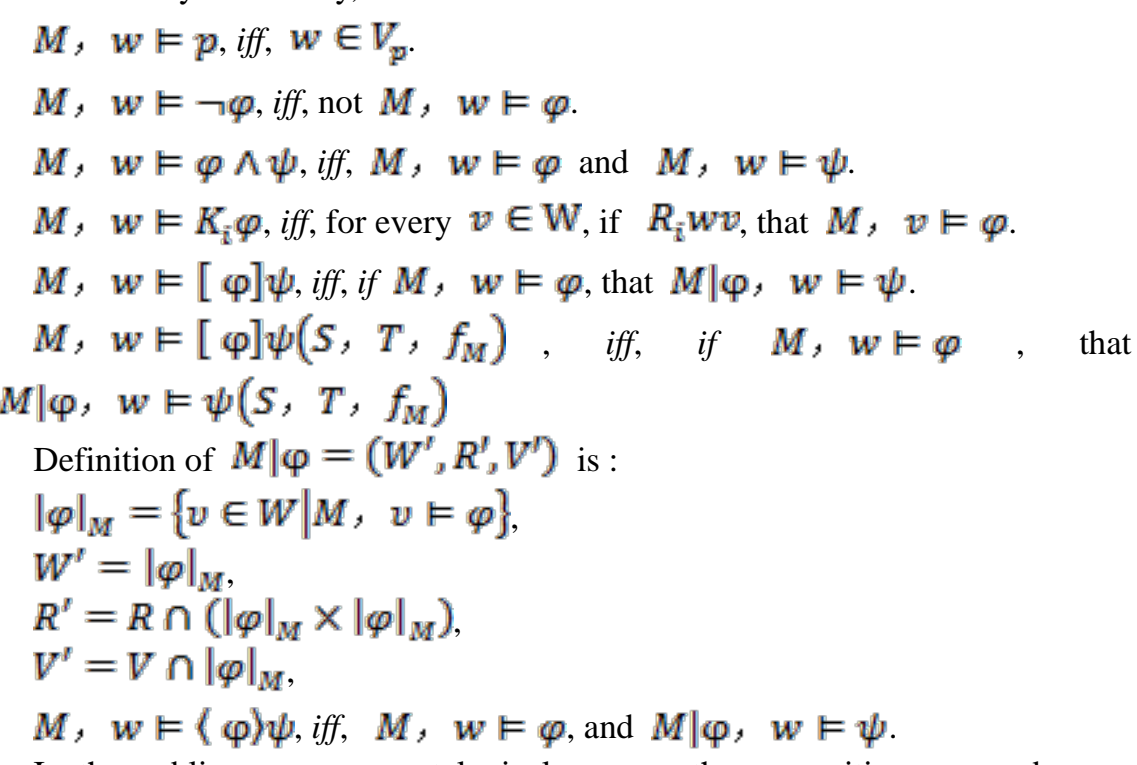

In the public announcement logic language, the proposition $\varphi$ can be any formula. From this, we cannot only to announce the knowledge of agent and the proposition which express the world itself, also can to announce the announcement itself. So the public announcement can be superimposed. For the listener, that means the superposition of listener's context and the metaphorical proposition by announced are announced to understand.

\section{Conclusion}

Through the above analysis, we can note that the dynamic logic description of metaphor understanding is hierarchical. First, speaker need to put forward metaphorical proposition by announcement, at the same time to update the old context set. After listener heard the announcement of the speaker, he understands it, and then announces his understanding as a proposition. On this basis, circling, 
the listener can become the speaker, the speaker maybe the listener, language communication activities are able to continue.

\section{References}

[1] G. Lakoff \& M. Johnson: Metaphor we live by. University of Chicago Press, Chicago, pp.3, (1980).

[2] H. V. Ditmarsch, W. V. der Hoek \& B. Kooi, Dynamic Epistemic Logic, Synthese Library Series, (2007).

[3]Zhou Chanle: The Computational Paraphrases for Chinese Metaphor, Beijing, Dongfang Press, 2009.

[4] Li Xiaowu \& He Chunxiu: An Epistemic Logic Characterizing Understanding, Journal of Southwest University (Social Science Edition), 5, pp.66-70, (2009).

[5] Kintsch, Walter. Metaphor comprehension: A computational theory, Psychonomic Bulltin \& Review, 7(2):257-266, 2000.

[6] Josef Stern. Metaphor in context. Mass: MIT Press, 2000.

[7] Carl Vogal, Dynamic Semantics for Metaphor, Metaphor and Symbol, 2001, 16(1\&2), 59-74.

[8] Zhou Chanle \& Zhang Wei: Study on Logical description of Chinese Metaphor Comprehension, journal of Chinese Information Processing, 2004(5). 